\title{
Non-invasive bladder volume measurement for the prevention of postoperative urinary retention: validation of two ultrasound devices in a clinical setting
}

\author{
Tammo A. Brouwer $^{1} \cdot$ Charina van den Boogaard ${ }^{2}$ Eric N. van Roon $^{3,4} \cdot$ Cor J. Kalkman ${ }^{5}$ Nic Veeger $^{6,7}$
}

Received: 20 October 2017 / Accepted: 23 February 2018 / Published online: 7 March 2018

(c) The Author(s) 2018. This article is an open access publication

\begin{abstract}
Ultrasound scanning of bladder volume is used for prevention of postoperative urinary retention (POUR). Accurate assessment of bladder volume is needed to allow clinical decision-making regarding the need for postoperative catheterization. Two commonly used ultrasound devices, the BladderScan ${ }^{\circledR}$ BVI 9400 and the newly released Prime ${ }^{\circledR}$ (Verathon Medical ${ }^{\circledR}$, Bothell, WA, USA), with or without the 'pre-scan' option, have not been validated in clinical practice. The aim of this study was to assess the performance of these devices in daily clinical practice. Between June and September 2016 a prospective observational study was conducted in 318 surgical patients (18 years or older) who needed a urinary catheter perioperatively for clinical reasons. For acceptable performance, we required that the volume as estimated by the BladderScan® differs by no more than 5\% from the actual urine volume after catheterization. The Schuirmann's two one-sided test was performed to assess equivalence between the BladderScan ${ }^{\circledR}$ estimate and catheterization. The BVI $9400 ®$ overestimated the actual bladder volume by $+17.5 \%$ (95\% CI +8.8 to $+26.3 \%)$. The Prime ${ }^{\circledR}$ without pre-scan underestimated by $-4.1 \%(95 \% \mathrm{CI}$ -8.8 to $+0.5 \%)$ and the Prime ${ }^{\circledR}$ with pre-scan underestimated by $-6.3 \%(95 \%$ CI -11.6 to $-1.1 \%)$. This study shows that while both ultrasound devices were able to approximate current bladder volume, both BVI $9400 \AA$ and Prime $®$-with and without pre-scan-were not able to measure the actual bladder volume within our predefined limit of $\pm 5 \%$. Using the pre-scan feature of the Prime ${ }^{\circledR}$ did not further improve accuracy.
\end{abstract}

Keywords Bladder catheterization · Bladder volume · Post operative urinary retention · Ultrasound BladderScan · Validation

Tammo A. Brouwer

t.brouwer@znb.nl

Charina van den Boogaard

charinavdboogaard@gmail.com

Eric N. van Roon

e.n.van.roon@znb.nl

Cor J. Kalkman

c.j.kalkman@umcutrecht.nl

Nic Veeger

nic.veeger@znb.nl

1 Department of Anaesthesiology, Medical Center Leeuwarden, Henri Dunantweg 2, PO Box 888, 8901 BR Leeuwarden, The Netherlands

2 Institute for Science, Medical Center Leeuwarden, Henri Dunantweg 2, PO Box 888, 8901 BR Leeuwarden,

The Netherlands
3 Department of Pharmacotherapy, Epidemiology and Economics, University of Groningen, Groningen, The Netherlands

4 Department of Clinical Pharmacy and Pharmacology, Medical Center Leeuwarden, Leeuwarden, The Netherlands

5 Division of Anesthesiology, Intensive Care and Emergency Medicine, University Medical Center Utrecht, Utrecht, The Netherlands

6 Clinical Epidemiologist, Department of Epidemiology, Medical Center Leeuwarden, Henri Dunantweg 2, PO Box 888, 8901 BR Leeuwarden, The Netherlands

7 Department of Epidemiology, University Medical Center, Groningen, The Netherlands 


\section{Introduction}

Transabdominal ultrasound is frequently used for measuring bladder volumes non-invasively to prevent postoperative urinary retention (= POUR) by timely catheterization, but also to avoid unnecessary bladder catheterizations [1-4]. Bladder catheterization is the "standard" treatment for POUR [1, 5, 6], but it is an invasive procedure that contributes to an increased risk of urinary tract infections, urethral trauma, patient discomfort and unplanned and prolonged hospital admissions [7-10]. Adequate monitoring of postoperative bladder volumes is mandatory to limit the risks associated with prolonged bladder overdistension or bladder catheterization $[1,2,11,12]$.

The BladderScan $®$ (Verathon $®$, Bothell, WA, USA) is a dedicated ultrasound device to clinically determine bladder volumes. The two latest editions of the BladderScan $\AA$ are the BVI 9400® and its successor the Prime $®$. Although both devices are CE marked and widely used in hospitals, the performance of their algorithms has never been validated in a perioperative clinical setting. The Prime ${ }^{\circledR}$ has the possibility to use a pre-scan function, showing real time echo images of the bladder next to the normally displayed scanned bladder. The aim of this prescan function is to improve accuracy.

The manufacturer of the BladderScan ${ }^{\circledR}$ claims an accuracy of $\pm 15 \% \pm 15 \mathrm{~mL}$. We, a priori, defined adequate clinical performance when the estimated volume differs from the actual urine volume after catheterization by no more than $5 \%$, based on clinical literature [13-15]. The aim of this study is to validate the performance of the BVI 9400® and the Prime $®$ (with and without pre-scan) in surgical patients by assessing the difference between the BladderScan ${ }^{\circledR}$ estimate and the actual urine volume assessed by catheterization.

\section{Methods}

\subsection{Design}

After institutional ethical approval, an investigator initiated prospective study was conducted between June and September 2016 in three groups of at least 100 consecutive surgical patients, each requiring bladder catheterization. All patients gave written informed consent. Bladder volumes were assessed using either the non-invasive BladderScan BVI $9400 \AA$ or the Prime $\AA$, with or without a pre-scan. Following the non-invasive estimation of the bladder volume all patients underwent urinary catheterization during which the actual urine volume was assessed as the "gold standard".

\subsection{Patient selection procedure}

Two groups of surgical patients (18 years or older, ASA classification I-IV), who required a perioperative bladder catheter following standard clinical hospital protocols, were included. The first group of patients consisted of surgical patients who needed a catheter after induction of anaesthesia and prior to the start of surgery due to expected volume shifts, epidural anaesthesia, or surgery in the smaller pelvis for example during coronary arterial bypass graft (CABG) surgery, orthopaedic hip or knee prostheses surgery or colonic surgery ("Perioperative Urinary Catheterization Protocol" Medical Center Leeuwarden). The second group of patients consisted of postoperative patients at the post anaesthesia care unit (PACU) who were unable to void spontaneously and needed a catheter to prevent bladder overdistension (bladder volume $\geq 500 \mathrm{~mL}$, "Micturition Protocol" Medical Center Leeuwarden). Exclusion criteria were a surgical incision in the suprapubic region, abdominal ascites, and pregnancy. Furthermore, surgical patients with an actual catheterized volume $\leq 30 \mathrm{~mL}$ in the operating theatre were excluded for analysis, as the BladderScan ${ }^{\circledR}$ is not sensitive to detect bladder volumes $\leq 30 \mathrm{~mL}$ (based on the information from the manufacturer).

\subsection{Sample size}

Based on previous studies we hypothesized that the estimated volume by the BladderScan ${ }^{\circledR}$ devices differs from the volume after catheterization by no more than $5 \%[13$, $15]$. With the predefined margin of equivalence of $\pm 5 \%$ and an assumed correlation of 0.90 , a sample size of at least 92 pairs per study group was needed to achieve $90 \%$ power to detect equivalence [16].

\subsection{Study procedure}

The researchers ( $\mathrm{CB}$ or $\mathrm{TAB})$ or operation nurses in the operating theatre and PACU performed all the measurements. They were trained in the use of the Prime ${ }^{\circledR}$ by an instructor of the manufacturer. Included patients with a clinical indication for intraoperative bladder catheterization were asked not to void shortly before the time of the surgery to ensure a measurable bladder volume. After induction of anaesthesia, three consecutive ultrasound measurements (scans) were performed in the supine position using the BladderScan ${ }^{\circledR}$ device in accordance with the cohort the patient was included. After three non-invasive measurements with each type of the BladderScan $\AA$, the patient was directly catheterized to measure the actual bladder volume. The first device to start with was the BVI9400®, by measuring bladder volumes non-invasively in 100 or more consecutive 
patients. After at least 100 patients were included with the BVI9400®, the next device used was the Prime ${ }^{\circledR}$ without pre-scan for measuring bladder volume in at least 100 consecutive patients. Again, after more than 100 patients were included with the Prime ${ }^{\circledR}$ without pre-scan, the Prime ${ }^{\circledR}$ with pre-scan function was used for measuring bladder volume non-invasively in at least 100 consecutive patients.

In included patients requiring postoperative bladder catheterization, the BVI $9400 ®$ was used at the PACU in accordance with the local hospital protocol (= postoperative bladder catheterization is indicated when the scanned bladder volume is larger than the threshold of $500 \mathrm{~mL}$ and the patient is unable to void spontaneously). If this was the case during the study period, then three consecutive measurements in the supine position were performed with the type of BladderScan ${ }^{\circledR}$ which was momentarily used in the intraoperative group of surgical patents measured in the OR, which were in following order; the BVI $9400 \AA$, the Prime ${ }^{\circledR}$ without pre-scan and the Prime ${ }^{\circledR}$ with pre-scan.

With the BVI $9400 \AA$ and Prime ${ }^{\circledR}$ without pre-scan, the aiming circle on the display was used to guide the position of the probe. In the Prime ${ }^{\circledR}$ with pre-scan, the "real echo" pre-scan image was developed to guide the position of the probe. By changing the position of the probe, a green line is trying to encircle the whole bladder echo image. If this was the case, this image could be fixed and a BladderScan was followed, assuming that the best image would lead to the best result $=$ measuring the total bladder with the largest bladder volume. A print of each measurement was collected to assess which of the three measurements was the "best" BladderScan ${ }^{\circledR}$ estimate, defined as the largest and most centrally displayed bladder together with the highest scanned volume. In all patients, catheterization took place immediately after the scanning procedure. The actual catheterized urine volumes were collected in a calibrated bowl. A $60 \mathrm{~mL}$ syringe was used to retrieve the last millilitres of urine to assess the precise volume and to be sure the bladder was completely emptied.

\subsection{Outcomes}

The primary outcome was the difference in volume in $\mathrm{mL}$ between the "best" estimated bladder volume measured with the BladderScan ${ }^{\circledR}$, using the three different methods (BVI $9400 \AA$, Prime ${ }^{\circledR}$ without pre-scan, and Prime $\AA$ with prescan) versus the actual measured urine volume after catheterization. In this, the difference between estimated and actual catheterized volume was expressed as the percentage of the actual catheterized volume.

Secondary outcomes included the difference in volume between the best estimated and the actual measured urine volume after catheterization over the small to large volume range ( $\leq 400$ and $>400 \mathrm{~mL}$ ). We choose $400 \mathrm{~mL}$, because volumes smaller than $400 \mathrm{~mL}$ are considered safe, not leading to bladder distention. Above $400 \mathrm{~mL}$, bladder volumes have to be measured non-invasively at regularly intervals to prevent bladder distention. In literature different volume limits are used for urinary retention $(400,500$ or $600 \mathrm{~mL})$ $[3,17,18]$. These commonly used volume limits are used to calculate the proportion of correct decisions to catheterize, after measuring the bladder volume non-invasively.

Furthermore, homogeneity of outcome in different patient subgroups was evaluated for gender, age, and BMI.

\subsection{Statistical analysis}

All data are expressed as mean \pm SD for normally distributed data and median with range for skewed data. Normality was assessed by visual inspection and the Shapiro-Wilk test.

For the primary outcome, the Schuirmann's two onesided test was used. For the secondary outcome, Bland-Altman analyses were performed to assess the level of agreement between the BladderScan ${ }^{\circledR}$ estimate and the actual volume after catheterization over the full range of volumes and over the two volume ranges of $\leq 400$ and $>400 \mathrm{~mL}$ [19].

To evaluate the performance of the devices with regard to correct clinical decision making the proportion true positives and true negatives were analysed. A Student's t test was used to evaluate differences between groups for normally distributed data, or a Mann-Whitney U test for non-normality. A one-way independent ANOVA or Kruskall-Wallis test was performed in case of a three-group comparison, depending on the normality of the data. For qualitative parameters, comparisons between groups were evaluated using Fisher exact test (dichotomous response) or Chi square test (exact when indicated). Preplanned subgroup analyses consisted of demographic characteristics that might influence the accuracy of the BladderScan ${ }^{\circledR}$ : gender (male vs. female), age ( $<60$ years vs. $\geq 60$ years), and BMI $\left(<25 \mathrm{~kg} / \mathrm{m}^{2}\right.$ vs. $25-30 \mathrm{~kg} / \mathrm{m}^{2}$ vs. $\geq 30 \mathrm{~kg} / \mathrm{m}^{2}$ ). A 2-tailed p-value less than 0.05 was considered statistically significant. All analyses were performed using commercially available computer software (Statistical Analysis System version 9.4, SAS Institute, Cary, NC, USA).

\section{Results}

Between June 2016 and September 2016, 392 consecutive patients were evaluated for participation in this study of which 345 were included. Of these, 318 patients were available for analysis (Fig. 1, Flow Chart).

Table 1 presents the demographic characteristics of the patients included. No statistically significant differences between the cohorts were observed at baseline, except for 


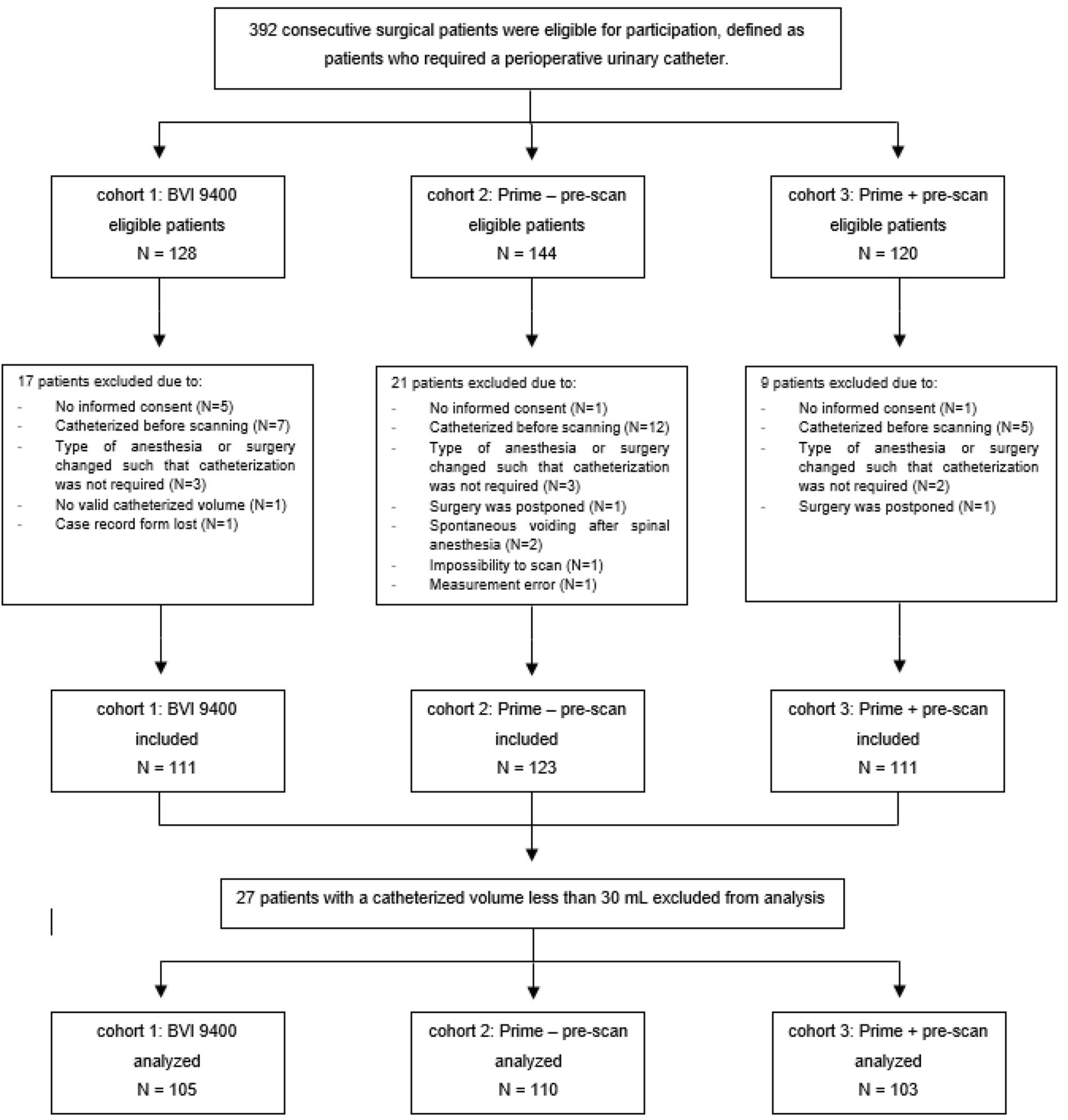

Fig. 1 Flow-chart patient selection

number of patients measured postoperatively. In the cohort of the BVI $9400 ®$ significant more patients were assessed postoperatively compared to the cohort of both Primes (15, 3 and $9 \%$ respectively, $p=0.003)$. In addition, measured bladder volumes (estimated and actual) are presented for all three cohorts.

\subsection{Primary outcome}

\subsubsection{Equivalence of bladder volume estimates}

For the primary endpoint of equivalence of bladder volume estimates, in all three cohorts the equivalence was not established. Over the whole volume range, the BVI $9400 \AA$ 
Table 1 Demographic characteristics

\begin{tabular}{lllll}
\hline Patient data & BVI 9400 $(\mathrm{n}=105)$ & $\begin{array}{l}\text { Prime without pre-scan } \\
(\mathrm{n}=110)\end{array}$ & $\begin{array}{l}\text { Prime with pre-scan } \\
(\mathrm{n}=103)\end{array}$ & p value \\
\hline Women no. (\%) & $52(49.5)$ & $42(38.2)$ & $47(45.6)$ & 0.23 \\
Age mean (SD) (year) & $65.9(13.4)$ & $66.6(12.5)$ & $64.5(12.4)$ & 0.41 \\
Age <60, no. (\%) & $31(29.5)$ & $26(23.6)$ & $29(28.2)$ & 0.61 \\
Weight (mean) $(\mathrm{kg})$ & $80.4(17.0)$ & $82.9(15.5)$ & $82.0(14.7)$ & 0.37 \\
Height (mean) $(\mathrm{cm})$ & $172.4(9.3)$ & $174.3(9.1)$ & $174.5(8.9)$ & 0.14 \\
BMI (mean) $\left(\mathrm{kg} / \mathrm{m}^{2}\right)$ & $27.0(4.8)$ & $27.2(4.2)$ & $27.0(4.6)$ & 0.88 \\
BMI <25 no. $(\%)$ & $38(36.2)$ & $40(36.4)$ & $38(36.9)$ & 0.97 \\
$\geq 25$ BMI <30 no. $(\%)$ & $44(41.9)$ & $45(40.9)$ & $39(37.9)$ & \\
BMI $\geq 30$ no. $(\%)$ & $23(21.9)$ & $25(22.7)$ & $26(25.2)$ & \\
Uterus no. $(\%)$ & $46(88.5)$ & $37(88.1)$ & $39(83.0)$ & 0.71 \\
\hline
\end{tabular}

$B M I$ body mass index overestimated the actual bladder volume by an average of $+17.5 \%(95 \% \mathrm{CI}+8.77$ to $+26.31, \mathrm{p}=1.00)$. The Prime ${ }^{\circledR}$ without pre-scan underestimated the actual bladder volume by an average of $-4.1 \%(95 \% \mathrm{CI}-8.78$ to $+0.49, \mathrm{p}=0.36)$ and the Prime ${ }^{\circledR}$ with pre-scan underestimated the actual bladder volume by an average of $-6.3 \%(95 \% \mathrm{CI}-11.57$ to $-1.07, \mathrm{p}=0.69)$ (Table 2).

\subsubsection{Level of agreement}

The BVI $9400 \AA$ overestimates the actual bladder volume with a bias of $+21.8 \mathrm{~mL}$ and limits of agreements (LOA) of $-99 \mathrm{~mL}$ to $+140 \mathrm{~mL}$ Both Prime ${ }^{\circledR}$ devices demonstrated a significant bias towards underestimation (both with and without performing a pre-scan, bias $=-20.7 \mathrm{~mL}$ ) and wide limits of agreement $(-183$ to $+141 \mathrm{~mL})$ (Fig. 2).

\subsection{Secondary outcomes}

\subsubsection{Subgroup analyses for homogeneity of BladderScan ${ }^{\circledast}$ performance}

As presented in Table 3, subgroup analysis for the homogeneity of the performance of the BladderScan ${ }^{\circledR}$ over different subgroups, showed that equivalence was not reached in any of the predefined subgroups. Although not reaching equivalence, some differences between subgroups were observed. For example, for the Prime ${ }^{\circledR}$ without and with pre-scan function, the underestimation in male patients was -0.8 and $+0.2 \%$ respectively, almost reaching equivalence $(\mathrm{p}=0.08)$. In patients with a $\mathrm{BMI}<25 \mathrm{~kg} / \mathrm{m}^{2}$ both Primes modes performed well, with an underestimation of -0.5 and $-2.7 \%$ respectively, but still not reaching equivalence $(\mathrm{p}=0.112$ and 0.266$)$.

For the BVI $9400 \AA$ used in patients with smaller or larger bladder volumes than $400 \mathrm{~mL}$ the overestimation decreased from $+22 \%$ in patients with an actual bladder volume $\leq 400 \mathrm{~mL}(\mathrm{~N}=79)$ to $+3.5 \%$ in patients with an actual bladder volume $>400 \mathrm{~mL}(\mathrm{~N}=26)$. In contrast, in the Prime ${ }^{\circledR}$ without pre-scan the underestimation increased from $-2.7 \%$ in patients with an actual bladder volume $\leq 400 \mathrm{~mL}(\mathrm{~N}=95)$ to $-13.1 \%$ in patients with an actual bladder volume $>400 \mathrm{~mL}(\mathrm{~N}=15)$. For the Prime ${ }^{\circledR}$ with pre-scan the underestimation increased from $-5.0 \%$ in patients with an actual bladder volume $\leq 400 \mathrm{~mL}(\mathrm{~N}=87)$ to $-13.6 \%$ in patients with an actual bladder volume $>400 \mathrm{~mL}$ $(\mathrm{N}=16)$.

Addressing the level of agreement using the Bland-Altman method showed the same results: the BVI 9400® showed less bias with bladder volumes $>400 \mathrm{~mL}$ (bias $=+10.0 \mathrm{~mL}$ ) than with smaller bladder volumes $\leq 400 \mathrm{~mL}$ (bias $=+25.7 \mathrm{~mL}$ ). For the Prime ${ }^{\circledR}$ device in both modes this was reversed: in patients with bladder volumes $\leq 400 \mathrm{~mL}$ the bias was -7.9 and $-4.0 \mathrm{~mL}$ and for patients with bladder volumes $>400 \mathrm{~mL}$ the bias was -101.5 and $-111.8 \mathrm{~mL}$ for the Prime ${ }^{\circledR}$ without and with pre-scan respectively (Table 4).

\subsubsection{Consequences for clinical decision-making}

With the BVI $9400 \AA$, the proportion true negative (=bladder volume measured and actual bladder volume both $\leq 500 \mathrm{~mL}$ ) and true positive (=bladder volume measured and actual bladder volume both $>500 \mathrm{~mL}$ ) clinical decisions whether or not to catheterize the bladder (using a threshold of $500 \mathrm{~mL}$ ) was 1.00 (95\% CI 0.96-1.00) and 0.91 (95\% CI 0.79-1.00), respectively. Comparable results were found with the Prime ${ }^{\circledR}$ without pre-scan 0.96 (95\% CI 0.92-1.00) and 0.86 (95\% CI 0.60-1.00) respectively and the Prime ${ }^{\circledR}$ with pre-scan 0.94 (95\%C I 0.87-0.98) and 1.00 (95\% CI $0.64-1.00$ ) respectively (Table 5). 
Table 2 Actual and estimated bladder volumes measured

\begin{tabular}{lcccc}
\hline & BVI 9400 (n=105) & $\begin{array}{l}\text { Prime with- } \\
\text { out pre-scan } \\
(\mathrm{n}=110)\end{array}$ & $\begin{array}{l}\text { Prime with Pre- } \\
\text { scan (n=103) }\end{array}$ & palue \\
\hline Estimated volume, mean (SD) (mL) & $288(237.0)$ & $191(163.7)$ & $203(182.1)$ & $<0.001$ \\
Actual volume, mean (SD) (mL) & $266(241.9)$ & $212(202.8)$ & $224(226.6)$ & 0.18 \\
Difference estimated-actual volume, & $21.8(59.9)$ & $-20.7(70.1)$ & $-20.7(82.7)$ & $<0.001$ \\
mean (SD) (mL) & & & $94(91.3)$ & 0.005 \\
Preoperative measurement, no (\%) & $89(84.8)$ & $107(97.30)$ & $16(15.5)$ & 0.078 \\
Actual volumes $>400 \mathrm{~mL}$, no (\%) & $26(24.8)$ & $15(13.6)$ & $14(13.6)$ & 0.194 \\
Actual volumes $>500 \mathrm{~mL}$, no (\%) & $17(16.2)$ & $9(8.2)$ & $8(7.8)$ & 0.149 \\
Actual volumes $>600 \mathrm{~mL}$, no $(\%)$ & $11(10.5)$ & $4(3.6)$ & &
\end{tabular}

\section{Discussion}

In this study, the performance of the BladderScan BVI $9400 \AA$ and its successor Prime ${ }^{\circledR}$ were assessed in surgical patients, both perioperative and postoperative. The estimates of bladder volumes were compared with actual bladder volumes collected after catheterization, which is considered the "gold standard" for measuring urinary volumes.

We a-priori set the margin of equivalence for a clinically acceptable estimation of the bladder volume to $\pm 5 \%$, based on the literature in which a mean difference of $-7 \%$ with the BVI $2500 \AA$ and a mean difference of $-3.3 \%$ with the BVI $3000 \AA$, an earlier version of the BladderScan, were observed $[13,15]$. In neither the BVI $9400 \AA$ nor the Prime ${ }^{\circledR}$ (with or without pre-scan option used) the performance was within our margin of equivalence. In this, the BVI $9400 \AA$ structurally overestimated the actual bladder volume and the Prime ${ }^{\circledR}$ without and with pre-scan structurally underestimated actual bladder volume. Underestimation may be preferred considering the fact that postoperative catheterization is a complication patients would like to avoid, but only if a strict "bladder protocol" is used to prevent bladder overdistension. Overestimation of the actual bladder volume will lead to earlier and more unnecessary postoperative urinary catheterizations [20].

When using the manufacturer's claim of an accuracy $( \pm 15 \% \pm 15 \mathrm{~mL})$ for the BVI $9400 \AA$ and the Prime ${ }^{\circledR}$, the amount of overestimation of the BVI 9400® was still beyond that level of accuracy. For the Prime ${ }^{\circledR}$ (both with or without pre-scan) the amount of underestimation was within the manufacturers' claim.

In clinical practice, a measured bladder volume of $500 \mathrm{~mL}$, which is considered a clinical cut-off value for bladder catheterization, should ideally not deviate more than 5\% from the true bladder volume (i.e., between 475 and $525 \mathrm{~mL}$ ), because in this range the BladderScan ${ }^{\circledR}$ can help the clinician in clinical decision-making. The manufacturer claims an accuracy of $\pm 15 \% \pm 15 \mathrm{~mL}$, both for the BVI $9400 \AA$ and the Prime $®$ (i.e. between 410 and $590 \mathrm{~mL}$ ). This is a rather large volume range, especially when the decision is whether or not to catheterize.

A possible explanation of these differences in the results between the BVI $9400 \AA$ (overestimating and performing better in bladder volumes $>400 \mathrm{~mL}$ ), and both Prime's (underestimating and performing better in bladder volumes $\leq 400 \mathrm{~mL}$ ) could be that the BVI $9400 \AA$ is using another, older algorithm, based on earlier devices such as the BVI $3000 \circledR$. The Prime ${ }^{\circledR}$ uses a new developed algorithm, which has only been tested in phantoms in the factory and not on patients.

When expressing the error in BladderScan ${ }^{\circledR}$ estimates in millilitres, using the Bland-Altman method, the mean bias was relatively small $(+22 \mathrm{~mL}$ for the BVI $9400 \AA$ and $-21 \mathrm{~mL}$ for both modes of the Prime $\left.{ }^{\circledR}\right)$. Previous studies investigating the accuracy of older models of the BVI (BVI $2500 \AA / 2500 \AA+$, BVI $3000 \AA)$ have reported similar mean errors (bias) ranging from -21.5 to $+19 \mathrm{~mL}$ [13-15]. However, the limits of agreement in both the BVI $9400 \AA$ and Prime ${ }^{\circledR}$ were large in bladder volumes $>400 \mathrm{~mL}$ (range $>340 \mathrm{~mL}$ for all three scanners) indicating a high variability between individual subjects towards both under- and overestimation. This was also reflected in the lack of equivalence in all three cohorts.

Additional subgroup analyses did not show significant differences between volumes $\leq 400$ and $>400 \mathrm{~mL}$. However, in patients with bladder volumes $>400 \mathrm{~mL}$ the BVI $9400 \AA$ tends to perform better with an average overestimation of $3.5 \%$ compared to $17.5 \%$ over the entire volume range. In the Prime ${ }^{\circledR}$ this was not observed; the average underestimation increased when measuring bladder volumes $>400 \mathrm{~mL}$ (to almost $-14 \%$ ). Although not reaching statistical significance, these results suggest that BVI $9400 \circledR$ estimates are more accurate in the higher volume range, whereas the Prime ${ }^{\circledR}$ appears to perform better in the lower volume range. Previous studies also reported higher discrepancies between the BladderScan ${ }^{\circledR}$ estimate and catheterization in actual bladder volumes of less than $50-100 \mathrm{~mL}$ and larger than $400 \mathrm{~mL}^{19}$. Nonetheless, in all 
a

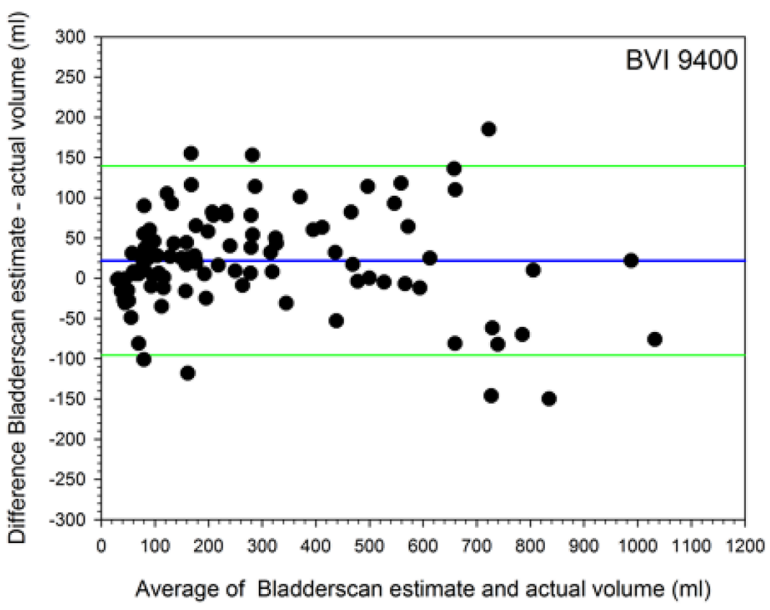

b

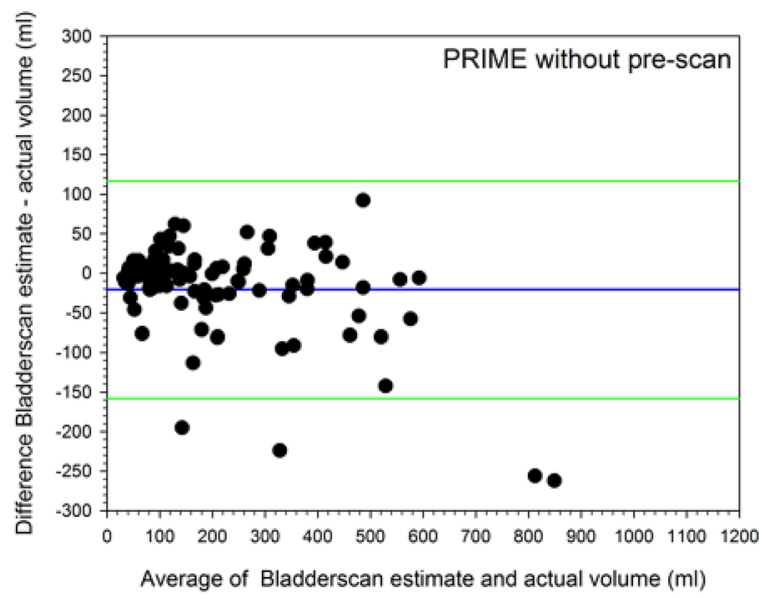

C

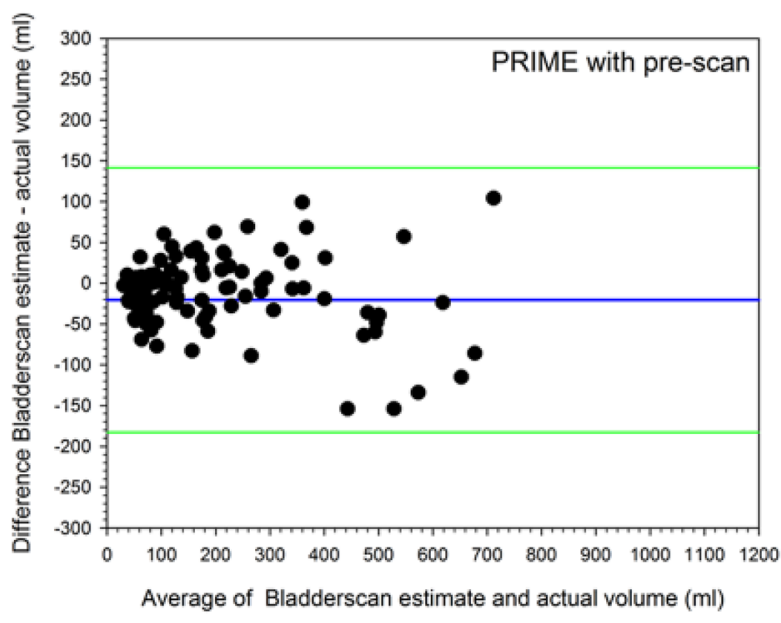

Fig. 2 a Bland-altman plot displaying the level of agreement of the BladderScan ${ }^{\circledR}$ Bvi 9400 over the whole volume range. b Bland-altman plot displaying the level of agreement of the prime ${ }^{\circledR}$ without pre-scan over the whole volume range. c Bland-altman plot displaying the level of agreement of the prime ${ }^{\circledR}$ with pre-scan over the whole volume range. Plotted is the average of the measured volume plus the scanned volume (X-axis), versus the difference of the measured volume minus the scanned volume (Y-axis). Blue horizontal line indicates the mean difference (=bias), and the green horizontal lines indicate two times standard deviation (= precision) three BladderScans the large variability did not have a strong impact on the occurrence of unnecessary (true positives $\geq 80 \%$ ) or missed (true negatives $\geq 94 \%$ ) catheterizations, although the numbers of patients with higher bladder volumes were too small to draw meaningful conclusions.

We expected that the Prime ${ }^{\circledR}$ with pre-scan ability would improve the accuracy of the measured bladder volume. Using real-time echo image should help to scan the whole bladder. In clinical practice this image was unsteady, and it was difficult to encircle the whole bladder with the green line. Therefore, the pre-scan function felt unreliable. It was surprising to find minimal differences between the Prime ${ }^{\circledR}$ without and with pre-scan. In fact, slightly better results were observed when using the Prime ${ }^{\circledR}$ without pre-scan, suggesting that using the prescan feature does not improve accuracy.

We also demonstrated that age or BMI did not significantly affect the accuracy of the BladderScan ${ }^{\circledR}$. However, with the Prime ${ }^{\circledR}$, we observed more accurate results in males. Previous studies also found that the BladderScan ${ }^{\circledR}$ (BVI $2500 \AA / 2500 \AA+$ ) was more accurate in males than females [13, 21], suggesting that the algorithm for female patients may need to be improved. Also, in patients with small BMI's $\left(<25 \mathrm{~kg} / \mathrm{m}^{2}\right)$ the BVI $9400 ®$ had difficulty in scanning the entire bladder (mean $+20.2 \mathrm{~mL}$ ) particularly with larger bladder volumes. The display of the device and its printouts showed that parts of the bladder were outside the scanning area with measured values labeled "larger than" $(>)$ instead of "is" $(=)$. Possibly, in lean patients the scanhead is positioned too close to the bladder, which prevents the device from scanning the whole bladder. This was not the case with the Prime®. In female patients and patients with a $B M I \geq 25 \mathrm{~kg} / \mathrm{m}^{2}$ these effects were less pronounced. Overall, our results suggest that the newer algorithms are more accurate in subgroups of patients (male patients and patients with a BMI $<25 \mathrm{~kg} / \mathrm{m}^{2}$ ), but still need improvement.

The strength of this study is that it was performed in a large number of unselected patients requiring bladder catheterization in a daily clinical practice setting. The same trained researcher $(\mathrm{CvdB})$ performed more than $80 \%$ of the measurements eliminating inter-observer bias. This might potentially limit the generalizability of our findings for settings where the measurements are performed by many different nurse operators - with varying experience in bladder ultrasound scanning. However, inter-observer reliability has been investigated in several previous studies and was found to be high [22-24]. Previous studies also showed that repeated measures do not improve the accuracy of the BladderScan ${ }^{\circledR}$ and that the results are independent of patient position and experience of the examiner [24, 25]. Another limitation of our study is that we did not include many patients with bladder volumes larger than $500 \mathrm{~mL}$. 
Table 3 primary and secondary outcomes and differences in percentages by patient characteristics and bladder volume $\leq 400$ and $>400 \mathrm{ml}$

\begin{tabular}{|c|c|c|c|c|c|c|c|c|c|}
\hline & \multicolumn{3}{|l|}{ BVI 9400} & \multicolumn{3}{|c|}{ Prime without pre-scan } & \multicolumn{3}{|c|}{ Prime with pre-scan } \\
\hline & Mean & $95 \% \mathrm{CI}$ & $\mathrm{p}$ & Mean & $95 \% \mathrm{CI}$ & $\mathrm{p}$ & Mean & $95 \% \mathrm{CI}$ & $\mathrm{p}$ \\
\hline Overall & $17.5(45.3)$ & 8.77 to 26.31 & 0.997 & $-4.1(24.5)$ & -8.78 to 0.49 & 0.357 & $-6.3(26.9)$ & -11.57 to -1.07 & 0.690 \\
\hline Male & $19.8(49.5)$ & 6.18 to 33.48 & 0.983 & $-0.8(24.5)$ & -6.71 to 5.16 & 0.080 & $0.2(25.6)$ & -6.67 to 7.03 & 0.082 \\
\hline Female & $15.2(41.0)$ & 3.80 to 26.61 & 0.961 & $-9.6(23.8)$ & -17.02 to -2.17 & 0.891 & $-14.1(26.5)$ & -21.85 to -6.261 & 0.998 \\
\hline Age $>60$ year & $16.4(33.9)$ & 3.95 to 28.82 & 0.964 & $-2.1(26.6)$ & -12.84 to 8.61 & 0.292 & $-7.2(25.7)$ & -16.93 to 2.61 & 0.673 \\
\hline Age $\leq 60$ year & $18.0(49.5)$ & 6.55 to 29.50 & 0.987 & $-4.8(24.0)$ & -9.98 to 0.44 & 0.465 & $-6.0(27.5)$ & -12.35 to 0.38 & 0.621 \\
\hline $\mathrm{BMI} \leq 25$ & $20.2(56.1)$ & 1.77 to 38.66 & 0.948 & $-0.5(22.8)$ & -7.83 to 6.74 & 0.112 & $-2.7(22.7)$ & -10.13 to 4.77 & 0.266 \\
\hline $25<\mathrm{BMI} \leq 30$ & $14.4(45.6)$ & 0.54 to 28.24 & 0.911 & $-6.4(25.2)$ & -13.99 to 1.12 & 0.648 & $-8.3(28.7)$ & -17.58 to 1.05 & 0.759 \\
\hline $\mathrm{BMI}>30$ & $19.1(17.9)$ & 11.39 to 26.90 & 0.999 & $-5.8(26.4)$ & -16.68 to 5.12 & 0.558 & $-8.7(30.0)$ & -20.82 to 3.40 & 0.733 \\
\hline$\leq 400 \mathrm{~mL}$ & $22.2(50.9)$ & 10.75 to 33.55 & 0.998 & $-2.7(25.1)$ & -7.86 to 2.38 & 0.191 & $-5.0(28.3)$ & -11.0 to 1.05 & 0.497 \\
\hline$>400 \mathrm{~mL}$ & $3.5(13.8)$ & -2.04 to 9.10 & 0.296 & $-13.1(18.6)$ & -23.32 to -2.78 & 0.943 & $-13.6(16.0)$ & -22.16 to -5.08 & 0.976 \\
\hline
\end{tabular}

Numbers in percentages $(\%)+$ standard deviation $(\mathrm{SD})$

Age in years

$B M I$ body mass index $\left(\mathrm{kg} / \mathrm{m}^{2}\right), C I$ confidence interval

Table 4 Bland-altman analysis over the two volume ranges $\leq 400$ and $>400 \mathrm{~mL}$ by type of BladderScan ${ }^{\circledR}$

\begin{tabular}{llllllll}
\hline & \multicolumn{2}{l}{ Volumes $\leq 400 \mathrm{~mL}$} & & \multicolumn{2}{l}{ Volumes $>400 \mathrm{~mL}$} & \\
\cline { 2 - 3 } & $\mathrm{N}$ & Bias & & LOA & $\mathrm{N}$ & Bias & LOA \\
\hline BVI 9400 & 79 & 25.7 & & -69 to 121 & 26 & 10.0 & -159 to 179 \\
Prime without pre-scan & 95 & -7.9 & & -81 to 65 & 15 & -101.5 & -385 to 182 \\
Prime with pre-scan & 87 & -4.0 & & -72 to 64 & 16 & -111.8 & -447 to 223 \\
\hline
\end{tabular}

Bias $=$ average of actual volume ${ }^{\wedge}$ scanned volume $(\mathrm{mL})$

LOA level of agreement $(\mathrm{mL})$

Table 5 The proportion well predicted clinical decisions whether or not to catheterize at a threshold of 400, 500, and $600 \mathrm{ml}$, by type of BladderScan ${ }^{\circledR}$

\begin{tabular}{|c|c|c|c|c|c|c|}
\hline & \multicolumn{2}{|l|}{$400 \mathrm{~mL}$} & \multicolumn{2}{|l|}{$500 \mathrm{~mL}$} & \multicolumn{2}{|l|}{$600 \mathrm{~mL}$} \\
\hline & True negatives & True positives & True negatives & True positives & True negatives & True positives \\
\hline BVI 9400 & $100(1.00-1.00)$ & $89.7(0.79-1.00)$ & $100(1.00-1.00)$ & $90.9(0.79-1.00)$ & $98.9(0.97-1.00)$ & $80.0(0.60-1.00)$ \\
\hline Prime without pre-scan & $97.9(0.95-1.00)$ & $87.5(0.71-1.00)$ & $96.1(0.92-1.00)$ & $85.7(0.60-1.00)$ & $98.1(0.96-1.00)$ & $100(1.00-1.00)$ \\
\hline Prime with pre-scan & $97.7(0.94-1.00)$ & $82.4(0.64-1.00)$ & $93.7(0.87-0.98)$ & $100(1.00-1.00)$ & $96.0(0.92-1.00)$ & $100(1.00-1.00)$ \\
\hline
\end{tabular}

Data are presented as percentage (95\% confidence interval)

Therefore, our findings with regard to the performance of the BladderScan ${ }^{\circledR}$ in the higher volume ranges and in correct clinical decision-making, need to be interpreted with caution.

\section{Conclusion}

This study showed that the ultrasound devices BVI $9400 \AA$ and Prime ${ }^{\circledR}$ with and without pre-scan were not able to measure the actual bladder volume within our strict predefined limits of $\pm 5 \%$. However, the BVI $9400 \AA$ performed better in bladder volumes $>400 \mathrm{~mL}$ and the Prime ${ }^{\circledR}$ performed better in bladder volumes $\leq 400 \mathrm{~mL}$, in male patients and in patients with a BMI $<25 \mathrm{~kg} / \mathrm{m}^{2}$. The pre-scan feature of the Prime ${ }^{\circledR}$ did not improve accuracy. Taken together, this suggests that the algorithms for measuring bladder volume non-invasively can be further improved, helping the clinician in making the right decision whether or not to catheterize, not too early (= unnecessary) but certainly not too late (= preventing bladder distention). An important lesson learnt is that new devices should be validated in clinical practice, on real patients, before they are sold to the market. On the other hand, despite their limited accuracy, the studied 
devices adequately facilitated clinical decision making when the decision is at hand whether it is necessary to catheterize the patient, especially when bladder volumes were $500 \mathrm{~mL}$ or more.

Author Contributions TAB Study design, patient recruitment, data collection, data analysis and interpretation, first author. CvdB Study design, patient recruitment, data collection, data analysis and interpretation, second author. ENvR Study design, analysis and interpretation data, critical revision of manuscript. CJK Study design, interpretation of data, critical revision of manuscript. NV Study design, data analysis and interpretation data, critical revision of manuscript.

Funding This work was supported by Verathon®, Global Headquarters Verathon Inc. 20001 North Creek Parkway, Bothell, WA 98011 USA. Financial support was used for financing the research assistant $(\mathrm{CvdB})$ and for data analysis.

\section{Compliance with ethical standards}

Conflict of interest Verathon® supplied the study with an unrestricted grant for financing the implementation of the study (research assistant, data collection and data analysis). Verathon $®$ had no influence on study design, on data collection and interpreting data, nor on the final draft or where to submit the paper. They trained medical personal in the BVI9400® and the Prime $®$ with and without prescan following the rules of using new Medical Technology in the Hospital (Dutch law). They were informed on the progress of the study. T.A. Brouwer, has received a travel grant for a clinical lecture about POUR for Verathon ${ }^{\circledR}$ sales marketers, but no other payments. Charina van den Boogaard, research assistant, was financial supported by the hospital. All other authors have no conflict of interest.

Open Access This article is distributed under the terms of the Creative Commons Attribution 4.0 International License (http://creativeco mmons.org/licenses/by/4.0/), which permits unrestricted use, distribution, and reproduction in any medium, provided you give appropriate credit to the original author(s) and the source, provide a link to the Creative Commons license, and indicate if changes were made.

\section{References}

1. Baldini G, Bagry H, Aprikian A, Carli F. Postoperative urinary retention: anesthetic and perioperative considerations. Anesthesiology. 2009;110:5:1139-57.

2. Darrah DM, Griebling TL, Silverstein JH. Postoperative urinary retention. AnesthesiolClin. 2009;27(3):465-84.

3. Brouwer TA, Rosier PF, Moons KG, Zuithoff NP, van Roon EN, Kalkman CJ. Postoperative bladder catheterization based on individual bladder capacity: a randomized trial. Anesthesiology. 2015;122(1):46-54.

4. Ozturk NK, Kavakli AS. Use of bladder volume measurement assessed with ultrasound to predict postoperative urinary retention. North Clin Istanb 2017 20;3(3):209-16.

5. Fitzpatrick JM, Desgrandchamps F, Adjali K, Gomez Guerra L, Hong SJ, El Khalid S, World Study Group, et al. Management of acute urinary retention: a worldwide survey of 6074 men with benign prostatic hyperplasia. BJU Int. 2012;109:1:88-95.

6. Halleberg Nyman M, Gustafsson M, Langius-Eklof A, Johansson JE, Norlin R, Hagberg L. Intermittent versus indwelling urinary catheterisation in hip surgery patients: a randomised controlled trial with cost-effectiveness analysis. IntJNursStud. 2013;50(12):1589-98.

7. Tammela T, Kontturi M, Lukkarinen O. Postoperative urinary retention. I. Incidence and predisposing factors. Scand J Urol Nephrol. 1986;20:3:197-201.

8. Tammela T, Kontturi M, Lukkarinen O. Postoperative urinary retention. II. Micturition problems after the first catheterization. Scand J Urol Nephrol. 1986;20(4):257-60.

9. Slappendel R, Weber EW. Non-invasive measurement of bladder volume as an indication for bladder catheterization after orthopaedic surgery and its effect on urinary tract infections. Eur J Anaesthesiol. 1999;16(8):503-6.

10. Sedor J, Mulholland SG. Hospital-acquired urinary tract infections associated with the indwelling catheter. Urol Clin North Am. 1999;26(4):821-8.

11. Carpenter FG. Impairment and restoration of rat urinary bladder responsiveness following distension. AmJPhysiol. 1983;244(1):R106-13.

12. Madersbacher H, Cardozo L, Chapple C, Abrams P, Toozs-Hobson $\mathrm{P}$, Young JS, et al. What are the causes and consequences of bladder overdistension? ICI-RS 2011. NeurourolUrodyn. 2012;31(3):317-21.

13. Brouwer TA, Eindhoven BG, Epema AH, Henning RH. Validation of an ultrasound scanner for determing urinary volumes in surgical patients and volunteers. J Clin Monit Comput. 1999;15(6):379-85.

14. Rosseland LA, Stubhaug A, Breivik H. Detecting postoperative urinary retention with an ultrasound scanner. Acta AnaesthesiolScand. 2002;46(3):279-82.

15. Byun SS, Kim HH, Lee E, Paick JS, Kamg W, Oh SJ. Accuracy of bladder volume determinations by ultrasonography: are they accurate over entire bladder volume range? Urology. 2003;62(4):656-60

16. Hinze SR, Wiley J. Testing the limits of testing effects using completion tests. Memory. 2011;19(3):290-304.

17. Pavlin DJ, Pavlin EG, Gunn HC, Taraday JK, Koerschen ME. Voiding in patients managed with or without ultrasound monitoring of bladder volume after outpatient surgery. Anesth Analg. 1999;89(1):90-7.

18. Lamonerie L, Marret E, Deleuze A, Lembert N, Dupont M, Bonnet $\mathrm{F}$. Prevalence of postoperative bladder distension and urinary retention detected by ultrasound measurement. Br J Anaesth. 2004;92(4):544-6.

19. Bland JM, Altman DG. Statistical methods for assessing agreement between two methods of clinical measurement. Lancet. 1986;1:(8476):307-10.

20. Medical Advisory Secretariat. Portable bladder ultrasound: an evidence-based analysis. Ontario Health Technol Assess Ser 2006; 6(11): $1-51$.

21. Huang YH, Bih LI, Chen SL, Tsai SJ, Teng CH. The accuracy of ultrasonic estimation of bladder volume: a comparison of portable and stationary equipment. Arch Phys Med Rehabil. 2004;85(1):138-41.

22. Marks LS, Dorey FJ, Macairan ML, Park C, DeKernion JB. Threedimensional ultrasound device for rapid determination of bladder volume. Adult Urol. 1997;50(3):341-8.

23. Massagli TL, Cardenas DD, Kelly EW. Experience with portable ultrasound equipment and measurement of urine volumes: inter-user reliability and factors of patient position. JUrol. 1989;142(4):969-71.

24. Ouslander JG, Simmons S, Tuico E, Nigam JG, Fingold S, BatesJensen B, et al. Use of a portable ultrasound device to measure post-void residual volume among incontinent nursing home residents. J Am Geriatr Soc. 1994;42(11):1189-92. 
25. Ding YY, Sahadevan S, Pang WS, Choo PW. Clinical utility of a portable ultrasound scanner in the measurement of residual urine volume. Singapore Med J. 1996;37(4):365-8. 